\title{
Nucleation in the presence of slow microscopic dynamics
}

\author{
Richard P. Sear ${ }^{\mathrm{a})}$ \\ Department of Physics, University of Surrey, Guildford, Surrey GU2 7XH, United Kingdom
}

(Received 6 March 2008; accepted 24 April 2008; published online 6 June 2008)

\begin{abstract}
Nucleation of a new thermodynamic phase is often a slow process due to the need to overcome a high free-energy barrier. However, there are other sources of slow dynamics; for example, at high densities/low temperatures, the movement of individual molecules or spins may be slow. Here, we study nucleation in a simple phenomenological model that has this type of slow microscopic dynamics. We do this to better understand how the two sources of slow dynamics interact. We find that as nucleation is intrinsically slow, only very slow microscopic dynamics strongly affect how nucleation occurs. The composition of the nucleus at the top of the nucleation barrier is much less sensitive to slow microscopic dynamics than is the composition of the nucleus once it is postcritical. However, slow dynamics affects not only the rate but also the pathway, which no longer goes over the saddle point in the free energy. We also find that the slow microscopic dynamics can cause sampling problems in an algorithm developed to calculate nucleation rates, and so cause it to predict the rate incorrectly. (C) 2008 American Institute of Physics. [DOI: 10.1063/1.2928844]
\end{abstract}

\section{INTRODUCTION}

Nucleation of a new thermodynamic phase is an activated process, and so it can be extremely slow. ${ }^{1,2}$ A stable phase is cooled, compressed, or otherwise moved into a state where it is no longer the equilibrium phase. There is then a thermodynamic driving force for the transformation to the equilibrium phase. This new phase can then form, starting off with a microscopic nucleus which grows into a bulk phase. However, due to the free-energy cost of the nucleus/bulk interface, the free energy initially increases as the microscopic nucleus of the equilibrium phase forms. Thus the free energy goes through a maximum; it is this maximum in the free energy that makes nucleation an activated process. The maximum in free energy is the free energy needed to create the nucleus at the top of the barrier $\Delta F^{*}$. The basic theory of nucleation is classical nucleation theory. It expresses the nucleation rate per molecule or per spin as the product of a kinetic prefactor $\nu$ and the exponential of the free-energy barrier $\Delta F^{*}, 1,2$

$$
\text { rate }=\nu \exp \left(-\Delta F^{*} / k T\right) .
$$

As the rate varies exponentially with $\Delta F^{*}$, attention is usually focused on this barrier. This is the probability that a nucleus is centered on any one molecule or spin. The prefactor is usually taken to be just one over a characteristic timescale, such as the time for a molecule or colloidal particle to diffuse its own diameter or for a spin to flip. The nucleation pathway is then assumed to be determined solely by the free-energy landscape; see Refs. 3-6 for examples. For example, the free energy of the nucleus of a binary mixture of components $A$ and $B$ may depend on two variables, the number of molecules of $A, N_{A}$, and the number of $B, N_{B}{ }^{7}$ Then the nucleus could in principle take any one of the many

\footnotetext{
a) Tel.: 44(0)1483-686793. FAX: 44(0)1483-686781. Electronic mail: r.sear@surrey.ac.uk.
}

pathways in the $N_{A}-N_{B}$ plane. However, if the pathway is determined by the free energy, then the nucleus will follow a path over the saddle point in the $N_{A}-N_{B}$ plane.

However, many systems possess slow microscopic dynamics. For example, recently Sanz et al. ${ }^{8}$ studied the crystallization of a model of a colloidal mixture of oppositely charged particles. They found that moves where a pair of oppositely charged particles swap places were very slow. These slow dynamics dramatically affect the nucleation dynamics: if these swaps are accelerated by changing their Monte Carlo kinetics, then a different crystal structure forms. Also, Schilling and Frenkel ${ }^{9}$ found that the crystallization of short rods was inhibited by the slow kinetics of rods rotating to align and form a second layer. A first layer formed swiftly but formation of the second was inhibited.

This situation, where some microscopic dynamics are fast but others are much slower, is precisely the situation we study here. We define a simple toy model that has these features and the simplicity of the model allows us to determine the nucleation pathway essentially exactly via direct simulations. This in contrast to earlier work where the techniques of umbrella sampling ${ }^{10}$ and forward flux sampling ${ }^{11-13}$ (FFS) were used. As we will see, these techniques may fail in the presence of slow microscopic dynamics.

So, there are two very different types of slow dynamics. There is nucleation, in which the slowness comes from the high free-energy cost of forming a critical nucleus that may have tens or hundreds of molecules or spins, and there is slow microscopic dynamics in which a motion of a single molecule or spin is very slow. Here we want to understand what happens when a system has both forms of slow dynamics. In particular, we want to better understand how nucleation occurs when the nucleus free energy is a function of two variables, one of which is very slow. The situation where both are very slow is trivial; this just corresponds to a very 
small $\nu$ in Eq. (1). Our two variables will be $n_{f}$, the number of fast spins in the nucleus, and $n_{s}$, the number of slow spins.

We will introduce a simple model in which we can continuously vary the speed of the slow variable. We will vary the rate of flips of the slow spins. We then study nucleation in this model and find that as we slow down the flips of the slow spins, the pathway of nucleation moves away from the saddle point. The nucleation pathway is then not determined by the form of the free energy alone; purely dynamic factors must also be taken into account. However, pushing the pathway away from the saddle point requires that the slow spin flips be very slow. As we move away from the saddle point, the free-energy factor in the nucleation rate, $\exp \left(-\Delta F^{*} / k T\right)$, decreases exponentially, so doing so is only favorable if the slow spin flips are exponentially slow. Thus the nucleation pathway is much less sensitive to slow dynamics than the postcritical growth of the new phase.

We also present the results of calculations using the FFS algorithm of Allen et al. ${ }^{11-13}$ This algorithm was developed to efficiently calculate the rates of barrier-crossing processes. We find that very slow dynamics cause the algorithm to sample poorly.

The next section describes both the free energy and dynamics of our model. We present and discuss our results in the third section, and the final section is a conclusion.

\section{MODEL}

We require a simple model both because we are interested in understanding the generic properties of nucleation in the presence of slow dynamics and because we need a model that is not computationally intensive in order to be able to compute the rate of an activated process via direct simulation. Thus we study nucleation where the nucleus has a simple phenomenological free energy.

\section{A. Phenomenological free energy}

The free energy is

$$
\begin{aligned}
& \Delta F\left(n_{f}, n_{s}\right)=\Delta F_{1}^{*}-c\left(n_{f}+n_{s}-n^{*}\right)^{2}-x n_{f} n_{s}, \\
& n_{f}, n_{s}=0,1,2, \ldots
\end{aligned}
$$

We will refer to $n_{f}$ and $n_{s}$ as the numbers of fast and slow spins, respectively. They are non-negative integer variables. By construction, along the $n_{f}$ and $n_{s}$ axes, there are equal nucleation barriers of height $\Delta F_{1}^{*}$. These barriers occur for nuclei of $n^{*}$ spins. The free energy $\Delta F$ we use in our calculations is plotted in Fig. 1.

The parameter $c=\Delta F_{1}^{*} /\left(n^{*}\right)^{2}$ so that the free energy $\Delta F=0$ at the origin. This leaves the parameter $x$. We use only $x>0$, for which there is a saddle point in the free energy $\Delta F$ in the $n_{f}-n_{s}$ plane. It is at $n_{f}^{*}=n_{s}^{*}=n^{*} /[2+x /(2 c)]$ and a free energy below that of the barriers along the $n_{f}$ and $n_{s}$ axes. Thus if the dynamics of our fast and slow spins are equal, nucleation occurs over this saddle point and along the line $n_{s}=n_{f}$. The saddle point free energy is

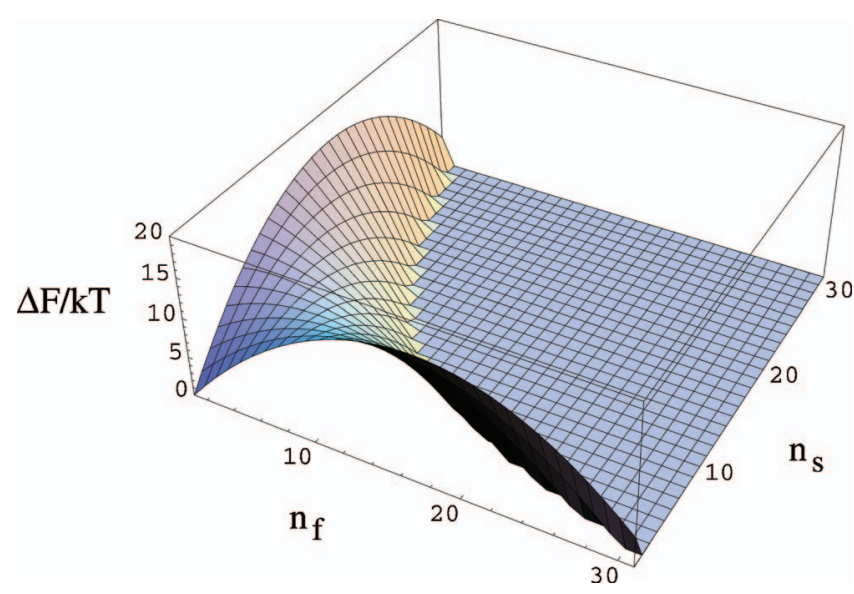

FIG. 1. (Color) Plot of the free energy of the nucleus, $\Delta F$, of Eq. (2) as a function of the number of fast spins, $n_{f}$, and the number of slow spins, $n_{s}$. Note that the free energy is symmetric around the line $n_{s}=n_{f}$. The parameter values are as follows: $\Delta F_{1}^{*}=16 k T$ and $x=0.15$. The free energy is cut off below 0 to improve the clarity of the plot. The saddle point is at $n_{s}=n_{f}=5$.

$$
\Delta F_{S}^{*}=\Delta F_{1}^{*}-\frac{\left(n^{*}\right)^{2} x}{4+x / c} .
$$

At all times, we will work at an on-axis nucleation barrier of $\Delta F_{1}^{*}=16 k T$ at $n^{*}=16$ spins and with $x=0.15$. Then the saddle point is $\Delta F_{S}^{*}=10 k T$ at $n_{f}=n_{s}=5$.

\section{B. Dynamics}

The free energy does not distinguish between fast and slow spins; exchanging the values of $n_{f}$ and $n_{s}$ does not change the free energy. However, the dynamics will in general distinguish between the fast and slow spins. The dynamics of the nucleus are simple. At each time, there are, by construction, four possible moves: adding a fast spin, losing a fast spin, gaining a slow spin, and losing a slow spin. The rates of these four moves are as follows:

$$
\begin{aligned}
& \overbrace{n_{f} \rightarrow n_{f}+1,}^{\text {move }} \overbrace{r_{+},}^{\text {rate }} \\
& n_{f} \rightarrow n_{f}-1, \quad r_{+} \exp \left(\left[\Delta F\left(n_{f}, n_{s}\right)-\Delta F\left(n_{f}-1, n_{s}\right)\right] / k T\right), \\
& n_{s} \rightarrow n_{s}+1, \quad r_{+} s \text {, } \\
& n_{s} \rightarrow n_{s}-1, \quad r_{+} s \exp \left(\left[\Delta F\left(n_{f}, n_{s}\right)-\Delta F\left(n_{f}, n_{s}-1\right)\right] / k T\right) \text {. }
\end{aligned}
$$

These dynamics preserve detailed balance. ${ }^{10}$ The characteristic timescales for the dynamics of fast and slow spins are $1 / r_{+}$and $1 /\left(r_{+} s\right)$, respectively. We will use $1 / r_{+}$as our characteristic timescale and measure all times in units of $1 / r_{+}$. The parameter $s \leqslant 1$ controls the relative speed of the dynamics of the slow spins. We simulate the nucleus using a standard Gillespie-type algorithm. ${ }^{14}$

\section{RESULTS}

Using the free energy and dynamics described above, we have calculated both nucleation rates and paths. In each case, we start with a nucleus with $n_{s}=n_{f}=0$ and take the nucleation time to be that to reach a point on the free-energy 


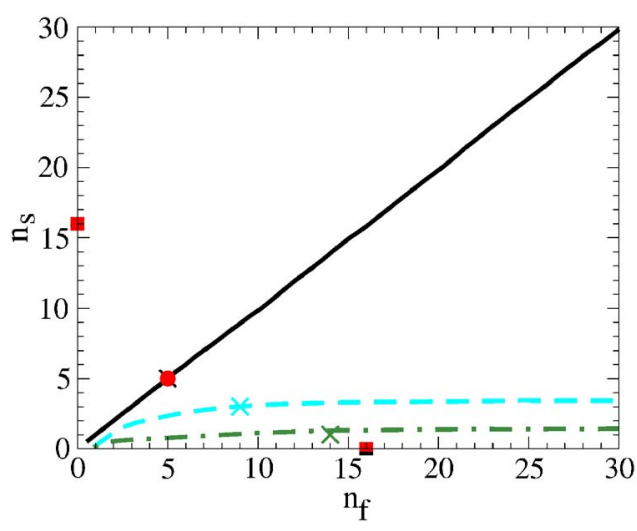

FIG. 2. (Color online) Plots of the averaged paths taken by nucleating clusters in the $n_{f}-n_{s}$ plane. The solid black, dashed cyan and dot-dashed green paths are for $s=1, s=10^{-3}$, and $s=10^{-6}$, respectively. The crosses on each curve are at the maximum in the free energy along each path. The saddle point is marked with a circle, and the tops of the nucleation barriers along the $n_{f}$ and $n_{s}$ axes one marked by squares.

surface well beyond the nucleation barrier. This is taken to be $n_{f}+n_{s}=60$. The nucleation rate is then 1 over the average of this time.

In Fig. 2, we have plotted averaged nucleation paths. We calculate an averaged path as follows. After each move of our simulation, we increment two counters. One counts the number of times our path samples each value of the nucleus size, $n_{f}+n_{s}$, and the other counts the fraction of fast spins at that particular value of the total nucleus size. The ratio of these counters gives the average fraction of fast spins as a function of the nucleus size, which we use to obtain the average paths in the $n_{f}-n_{s}$ plane in Fig. 2.

If $s=1$, then the fast and slow spins are completely equivalent and we expect nucleation to occur very close to equilibrium, i.e., the nucleus will always be close to its minimum free energy which is along the line $n_{s}=n_{f}$. On this line, the barrier height for nucleation is a minimum. The black curve in Fig. 2 is the path for $s=1$. We see that indeed the path goes through the saddle point at $n_{f}=n_{s}=5$ and that it lies along the line $n_{s}=n_{f}$ where the free energy is lowest. Thus, for $s=1$, the situation is straightforward. The free energy dominates and dictates that nucleation occurs over the saddle point in the free-energy landscape.

The other extreme is $s=0$. Then the cluster that forms is purely composed of fast spins; $n_{s}=0$ at all times. The cluster is now forced to move along the $n_{f}$ axis in Fig. 1. Along this axis, the barrier to be overcome is $\Delta F_{1}^{*}=16 k T$, which is $6 k T$ higher than that at the saddle point. This barrier increase is predicted to slow down nucleation by a factor of $\exp (6)$ $\simeq 400$. Our calculations yield nucleation rates of $5 \times 10^{-5} r_{+}$ for $s=1$ and $1 \times 10^{-8} r_{+}$for $s=0$. This is a slow down by a factor of 5000. A simple estimate based on the change in the barrier height underpredicts the difference. This is possibly due to the fact that for $s=0$, there is only one path, that with $n_{s}=0$, while for $s=1$, paths that do not precisely go over the saddle point may still contribute to and so increase the rate.

Having considered the two extremes, let us consider intermediate values of $s$, i.e., less than 1 but nonzero. Then the nucleus will contain slow spins but then there is a trade-off between having more slow spins and so a lower free energy

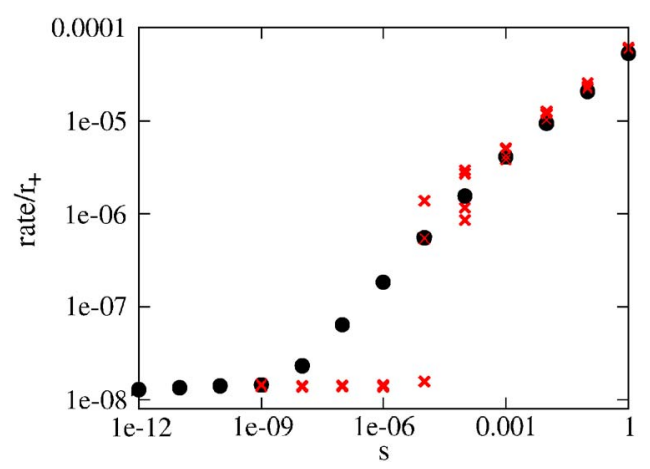

FIG. 3. (Color online) Plots of the nucleation rate as a function of the slowness of the slow spin dynamics, $s$. The black circles are the essentially exact results of direct simulations. The error bars on these rates are approximately equal to the size of the symbols. The crosses are the results of FFS calculations with $n_{c}=10000$ configurations. For each value of $s$, we plot the results of four independent runs.

but having to wait longer for more slow spins to flip. The nucleation rate is plotted as a function of the slowness of the slow spin dynamics in Fig. 3. We see that the nucleation rate decreases with decreasing $s$. It does so over a very large range of values of $s$. Note that in Fig. 3, the rate only plateaus for $s<10^{-8}$, comparable to the nucleation rate in the $s=0$ limit. This is because until $1 /\left(r_{+} s\right)$ is much larger than the time taken to surmount the barrier along the $n_{f}$ axis, $\Delta F_{1}^{*}$, it is still favorable for the nucleus to have at least one or two slow spins. Unless the time to flip a single slow spin $1 / \mathrm{s}$ $\gg \exp \left(\Delta F_{1}^{*} / k T\right)$, the nucleus will typically have at least one slow spin, and so nucleation will not occur directly along the $n_{f}$ axis and the rate will depend on the value of $s$.

The process of growth is much faster than that of nucleation. In postcritical growth, the nucleus gains spins at a rate of order $r_{+}$. Therefore, as soon as $s \ll 1$, the growing postcritical nucleus is far from equilibrium. It grows almost entirely by gaining fast spins. This is clearly seen in Fig. 2, where we see that once the path for $s=10^{-3}$ (cyan) has passed the top of the barrier (marked by a cross), it is almost horizontal. Further decreases in $s$ then have little effect on growth.

\section{A. Results of FFS calculations}

Here we apply the FFS technique of Allen et al. ${ }^{11-13}$ to calculate a nucleation rate. This is one of the techniques used to calculate the nucleation rate when it is too small to be calculated via direct simulation. ${ }^{2}$ As we have calculated the rate essentially exactly via direct simulation, we can compare the results of FFS calculations to exact results to see whether or not the FFS technique can cope with the slow dynamics. The FFS results are shown as red crosses in Fig. 3. Each point is the result of a FFS calculation in which $n_{c}=10000$ configurations are stored at each interface. We plot the results of four independent runs for each value of $s$ in order to show the scatter in the results. The order parameter used is $n_{T}=n_{s}+n_{f}$. The first interface (used for the flux calculation) is at $n_{T}=2$ and subsequent interfaces are at values of $n_{T}$ $=4,6,8, \ldots$. See Refs. 11-13 for details of the technique.

We see that for $s \gtrsim 10^{-3}$, the FFS calculation with 10000 stored configurations gives a good estimate of the rate. It also 
gives the $s=0$ limit of the rate correctly. For $s \gtrsim 10^{-3}$ there is a systematic slight overestimate of the rate, which may be due to FFS neglecting the time the system spends actually on the barrier. This is minor. However, for $s=10^{-4}$ and $10^{-5}$, the scatter in the runs is very large, while for $s=10^{-6}$, FFS predicts a rate that is already in the $s=0$ limit. This is incorrect; in fact, it is approximately an order of magnitude higher. Examination of the paths FFS is sampling (not shown) show that here FFS is not sampling the paths correctly; it samples paths that lie too close to the $n_{f}$ axis. From Fig. 3 , it is clear that when $s n_{c} \lesssim 1$, the FFS sampling is poor, leading to large scatter, whereas for $s n_{c} \ll 1$, the FFS technique completely misses the $n_{s} \neq 0$ paths, and so predicts, with little scatter, an incorrect rate. We have also performed simulations with $n_{c}$ $=100$ (not shown) and then the point where the scatter in the FFS rates becomes very large is moved to larger values of $s$.

Let us return to the findings of Sanz et al. ${ }^{8}$ and consider them in the light of our results for FFS. In their system, the slow dynamics is the swapping of charged particles of opposite charges. If we denote the natural frequency of such swaps by $f$, then if $n_{c} f \ll 1$, where $n_{c}$ is their number of configurations at an interface, we can easily understand their finding that the FFS algorithm sampled a pathway that proceeds without these slow swaps. If a move such as a swap occurs with a very low frequency, a simulation simply will not sample it. This is just like the situation with the current model with, say, $s=10^{-6}$ and $n_{c}=100$. From Fig. 2, we see that the true nucleation path for this value of $s$ has approximately one slow spin. On average, it will take $10^{6}$ steps for the nucleus to acquire one slow spin, but of course, if our FFS simulation is only working with $n_{c}=100$ configurations and the runs from interface to interface take only a few steps, the simulation may never sample a single slow spin flip and so miss the true nucleation pathway.

\section{CONCLUSION}

We have found that slow microscopic dynamics can change not only the nucleation rate but also its pathway. In our simple model, the nucleation rate dropped by more than three orders of magnitude as the slow microscopic dynamics slowed down by nine orders of magnitude. In other systems with larger nucleation barriers, we expect even larger reductions in the nucleation rate over even larger ranges in the speed of the slow variable. In the presence of slow microscopic dynamics, the pathway for nucleation will not in general go over the saddle point in the free-energy landscape. However, as nucleation is itself an intrinsically slow process, the pathway is less sensitive to the speed of the slow dynamics before the nucleation barrier than afterwards, when the nucleus is growing rapidly. For example, we saw in Fig. 2 that for $s=10^{-3}$, the composition of the critical nucleus is not the equilibrium one: $n_{f} / n_{s} \simeq 3$ not 1 as it is at the saddle point. However, once the nucleus has surmounted the barrier, the pathway is almost horizontal: the composition moves dramatically towards being almost pure fast spins, $n_{f} / n_{s} \gg 1$, and so is very far from the equilibrium composition.

Most of what we know about the molecular details of nucleation we have learnt from simulation. ${ }^{2}$ Quantitative calculation of the nucleation rate usually requires the use of specialized techniques such as the umbrella sampling technique ${ }^{10}$ used by Schilling and Frenkel ${ }^{9}$ or FFS. ${ }^{8,11-13}$ We have seen that FFS may fail when there are slow microscopic dynamics. These cause sampling problems that mean the nucleation pathways are not correctly sampled and hence the nucleation rate is not calculated correctly. In addition, with umbrella sampling, even if the complete free-energy landscape is obtained, as the nucleation pathway is not solely determined by the free energy, this is not enough to calculate the rate. In conclusion, a straightforward application of existing techniques may not be enough to accurately calculate nucleation rates in these systems and so further developments may be required.

\section{ACKNOWLEDGMENTS}

It is a pleasure to acknowledge stimulating discussions with R. Allen, D. Frenkel, A. Page, E. Sanz, and C. Valeriani.

${ }^{1}$ P. G. Debenedetti, Metastable Liquids (Princeton University Press, Princeton, 1996).

${ }^{2}$ R. P. Sear, J. Phys.: Condens. Matter 19, 033101 (2007).

${ }^{3}$ P. R. ten Wolde and D. Frenkel, Science 277, 1975 (1997).

${ }^{4}$ V. Talanquer and D. W. Oxtoby, J. Chem. Phys. 109, 223 (1998).

${ }^{5}$ P. Welch and M. Muthukumar, Phys. Rev. Lett. 87, 218302 (2001).

${ }^{6}$ D. Moroni, P. R. ten Wolde, and P. G. Bolhuis, Phys. Rev. Lett. 94, 235703 (2005).

${ }^{7}$ M. E. McKenzie and B. Chen, J. Phys. Chem. 110, 3511 (2006).

${ }^{8}$ E. Sanz, C. Valeriani, D. Frenkel, and M. Dijkstra, Phys. Rev. Lett. 99, 055501 (2007).

${ }^{9}$ T. Schilling and D. Frenkel, Phys. Rev. Lett. 92, 085505 (2004).

${ }^{10}$ D. Frenkel and B. Smit, Understanding Molecular Simulation (Academic, New York, 2001).

${ }^{11}$ R. J. Allen, P. B. Warren, and P. R. ten Wolde, Phys. Rev. Lett. 94, 018104 (2005).

${ }^{12}$ R. J. Allen, P. B. Warren, and P. R. ten Wolde, J. Chem. Phys. 124, 024102 (2006).

${ }^{13}$ R. J. Allen, D. Frenkel, and P. R. ten Wolde, J. Chem. Phys. 124, 194111 (2006).

${ }^{14}$ D. T. Gillespie, J. Phys. Chem. 81, 2340 (1977). 